\title{
MPPT OF PHOTOVOLTAIC SYSTEM VARIABLE ACCELERATION DISTURBANCE METHOD BASED ON GENETIC ALGORITHM
}

\author{
Shengqing Li, ${ }^{*}$ Wenfeng $\mathrm{Wu},{ }^{*}$ and Dinghuan $\mathrm{Ma}^{*}$
}

\begin{abstract}
The benefits of PV have limited its large-scale development. To improve the efficiency of photovoltaic power generation, a PV Maximum Power Point Tracking (MPPT) accelerating perturbation method based on genetic algorithm is proposed by analysing the mathematical model of PV cells and optimizing MPPT controller strategy. The difference between the variable step perturbation method and the variable accelerating perturbation method is compared, and the acceleration process of the variable accelerating perturbation method is proved by mathematical expression. To further improve the tracking accuracy and reduce the tracking time of the system, the genetic algorithm is used to establish the initial search range. Finally, the simulation model is built in Matlab/Simulink software platform, and the correctness of the method is verified by simulation.
\end{abstract}

\section{Key Words}

MPPT, photovoltaic system, genetic algorithm, acceleration disturbance

\section{Introduction}

As one of the cleanest energy sources, solar energy has been widely used in life production. The solar power system is highly valued by countries and has become a hot new energy industry because of its safety, convenience, and cleanliness. However, due to the low efficiency and high cost the development of photovoltaic power generation has been limited until now. At present, over all the countries, scholars are committed to improving the efficiency of photovoltaic power generation system. In photovoltaic power generation system, the Maximum Power Point Tracking (MPPT) controller determines the size of the output power.

\footnotetext{
* College of Electrical and Information Engineering, Hunan University of Technology, Zhuzhou, Hunan, 412007, China; e-mail: lsq1961@sohu.com, wuwenfeng1992@163.com, 624562521@qq.com

Recommended by Prof. Anmin Zhu
}

(DOI: 10.2316/Journal.206.2018.2.206-5131)
Due to the characteristics of the internal structure, the photovoltaic cell is subject to the influence of light intensity and temperature, and the output characteristic curve has strong nonlinear characteristics [1]. Research on MPPT technology in recent decades, the MPPT controller strategy from the initial method, such as Incremental Conductance (INC), Constant Voltage (CV) Method, and Perturbation and Observation ( $\mathrm{P} \& \mathrm{O}$ ) Method, is derived from different methods [2]-[6].

For the traditional $\mathrm{P} \& \mathrm{O}$ power consumption, easy to cause the shortcomings of oscillation Yang et al. [7] have proposed a variable step-size control method to overcome the shortcomings of the traditional perturbation method, by selecting different steps according to the different power variation, which reduces the power loss due to voltage variation. However, it cannot make quick judgments in the case of the changes in light intensity. Hang et al. [8] have proposed a variable step size weak oscillation perturbation method in which the accuracy of the system is improved, and the oscillation of the system is eliminated. However, the two-step step makes the tracking speed of the system lower. In recent years, with the development of intelligent algorithms, and in the parade control to show more and more advantages, many international scholars have applied the intelligent algorithm to the PV MPPT [9]-[11]. The method of combining traditional perturbation method with particle swarm optimization is proposed [9], in which the MPPT is divided into two steps: the local maximum point is searched by the fixed-step perturbation method, and the particle swarm optimization algorithm is used to find the global maximum point. This method improves the convergence rate by reducing the particle swarm algorithm time, however, there are still a lot of shock near the maximum power point (MPP), so the stability of the system needs to be improved. Mohamed et al. [11], by combining fuzzy logical (FL) control, genetic algorithm (GA) and small-signal analysis, proposed a new type of PV system MPPT controller technology, in which using GA for tuning FL system parameters reduces time consumption, however, the oscillation near the MPP diminished is not obvious. To reduce the oscillation at the MPP, this paper 
improves the variable step perturbation method and to reduce the tracking time, this paper uses GA for intelligent search.

\section{Principle Analysis}

The photovoltaic grid-connected power generation system model shown in Fig. 1 mostly consists of four parts, which are PV array, MPPT controller, inverter, and grid.

\subsection{Photovoltaic Cell Model}

The working principle of photovoltaic cells is the photoelectric effect. By solar radiation, the radiation energy is converted into electrical energy. According to the internal structure of photovoltaic panels, in engineering application, the working principle of the equivalent circuit is shown in Fig 2 [12].

In Fig. $2 I_{p h}$ is the output current for photovoltaic battery, $I_{o}$ is the diode reverse saturation current, $R_{s h}$ and $R_{s}$, respectively, represent the internal equivalent of photovoltaic cells in parallel and series resistance, and $I$ and $U$ respectively, represent the output current and voltage of the photovoltaic circuit.

The mathematical model of the photovoltaic cells can be expressed as follows:

$$
I=I_{p h}-I_{o}\left\{\exp \left[\frac{q\left(U+R_{s} I\right)}{n K T}\right]-1\right\}-\frac{U+R_{s} I}{R_{s h}}
$$

Taking solar $(S)$ and temperature $(T)$ into consideration, we have:

$$
\left\{\begin{array}{c}
I_{o}=I_{r}\left[\frac{T}{T_{r}}\right]^{3} \exp \left(\frac{q E_{G}}{K n T}\left[\frac{1}{T_{r}}-\frac{1}{T}\right]\right) \\
I_{p h}=\left[I_{s c}+k_{1}\left(T-T_{r}\right)\right] \frac{S}{100}
\end{array}\right.
$$

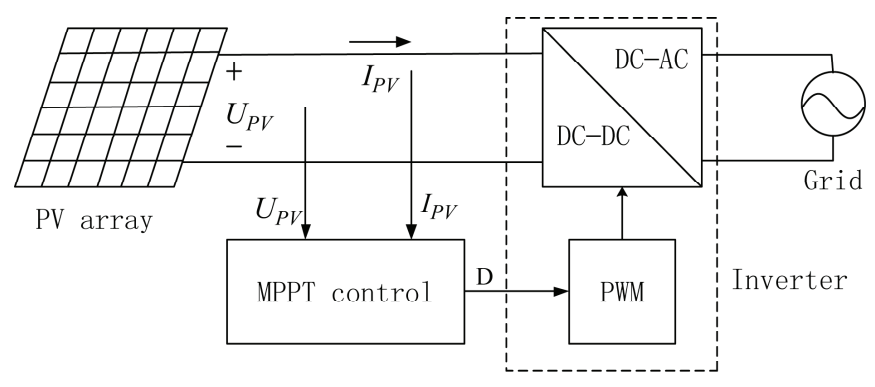

Figure 1. Photovoltaic power generation system model.

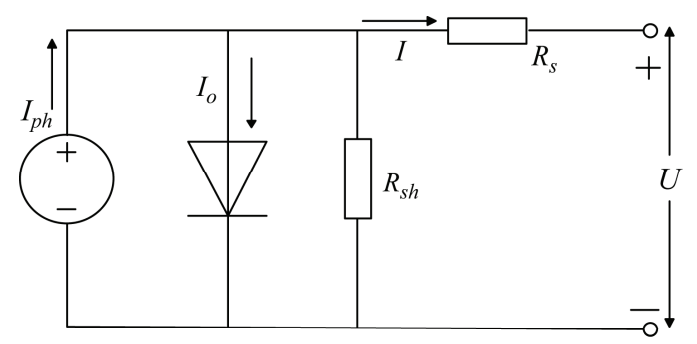

Figure 2. Equivalent circuit of photovoltaic cell.
In (1) and (2), $q$ is the charge quantity with its value being $1.6029 \times 10^{-19} \mathrm{C}, K$ is the Boltzmann constant that is concerned with temperature and energy with its value being $1.3819 \times 10^{-23} \mathrm{~J} / \mathrm{K}, U$ is the no-load voltage for the battery, $E_{G}$ is the energy function of semiconductor materials in batteries, $T_{r}$ is the reference temperature, $I_{r}$ is the current at the reference temperature, $I_{s c}$ is the short-circuit current for the reference condition, $k_{1}$ is the temperature coefficient of short circuit current, and $n$ is the diode fitting coefficient.

From (1) and (2), it can be seen that the output current $I$ of the photovoltaic circuit has a complex nonlinear relationship with $T$ and $S$. Due to the large $R_{s h}$, so in practice, the impact of $R_{s h}$. In practice, the $R_{s h}$ is too large to ignored, thus the mathematical model of a single block of photovoltaic cells' output current is in the form of:

$$
I=I_{p h}-I_{o}\left\{\exp \left[\frac{q\left(U+R_{s} I\right)}{n K T}\right]-1\right\}
$$

\subsection{MPPT Working Principle}

Under standard conditions $\left(T=25^{\circ} \mathrm{C}, S=1,000 \mathrm{~W} / \mathrm{m}^{2}\right)$, photovoltaic cells output power and voltage characteristics curve shown in Fig. 3. It can be seen that point $M$ corresponds to the maximum power, let the voltage of point $\mathrm{M}$ be $U_{\mathrm{M}}$, the power of point $\mathrm{M}$ be $P_{\mathrm{M}}$, then the voltage on both sides of the $\mathrm{M}$ point corresponding to the power are less than $P_{\mathrm{M}}$. To improve the power generation rate of photovoltaic power generation system, it is necessary to make the PV array output power always at the MPP or near the MPP [13]-[15].

The segmental analysis of characteristic curves is as follows: in the A-B and $\mathrm{C}-\mathrm{D}$ segments, the output power $P$ changes more obviously with the voltage $U$ (approximately linear change); in the $\mathrm{B}-\mathrm{C}$ region, this trend is decreasing; and especially in the region of $\mathrm{E}-\mathrm{F}$, the power varies little with voltage. The classical perturbation method changes the output voltage of the PV array according to the fixed step size, thereby changing the duty cycle of the DC-DC converter, while the variable step perturbation method according to the PV array conductivity changes, thereby selecting the appropriate step to change the output voltage

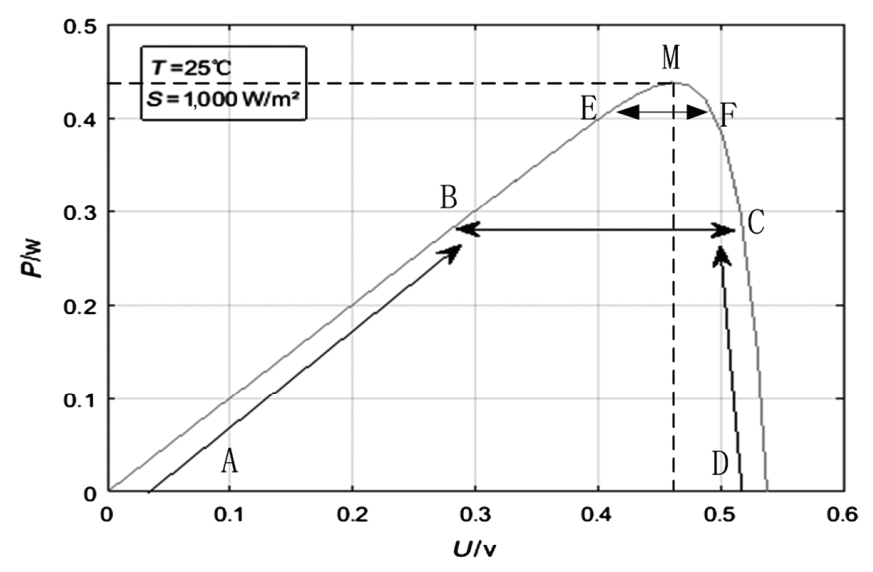

Figure 3. $P-U$ characteristic curve. 
[16]-[18]. The two methods produce oscillations in the region of $\mathrm{E}-\mathrm{F}$. Compared with the traditional perturbation method, the oscillation of the variable step perturbation method near the MPP is reduced, and the tracking time is also reduced. As the regions $\mathrm{A}-\mathrm{B}$ and $\mathrm{C}-\mathrm{D}$ show "linear change", a variable acceleration disturbance method based on GA is presented in this paper, the GA is used to search the two intervals to establish a more accurate search range, while the improved variable step disturbance method is used to search for the $\mathrm{B}-\mathrm{C}$ region to reduce the oscillation and tracking time.

\section{Optimization of MPPT Controller Strategy}

\subsection{Step Optimization}

The variable step perturbation method [19] selects the step size adjustment factor update rule as follows:

$$
\begin{aligned}
& D_{e}(k)=\frac{1}{I(k)}\left|\frac{d P}{d U}\right|=1+\frac{U(k)}{I(k)}\left|\frac{d I}{d U}\right| \\
& U_{\text {ref }}(k)=U_{\text {ref }}(k-1) \pm D_{e}(k) \Delta U_{\text {ref }}
\end{aligned}
$$

where $X$ represents the power, voltage and current. In (5), $\Delta U_{\text {ref }}$ is the fixed step perturbation, $U_{r e f}(k)$ is the reference voltage value of time $k$, and $U_{r e f}(k-1)$ is the reference voltage value of time $k-1$.

This method does not take full account of the difference in tilt angle between the BM segment and the CM segment, that is, the inclination angle of the BM segment is less than that of the CM segment. If the same standard is used to disturb, then the CM section takes more time. Therefore, this paper proposes a segmented acceleration perturbation method, which is based on the value of $d U$, dividing the disturbance into the following cases:

1. When $|d U| \leq \varepsilon$ and $|d I| \leq \mu$

When $|d U| \leq \varepsilon$ and $|d I| \leq \mu$, which means $|d P| \leq e_{0}$, $U(k+1)=U(k)$ and $I(k+1)=I(k)$. As $|d P|=$ $|d U \cdot d I| \leq \varepsilon \cdot \mu$ is a very small range, it can be considered that this point is the MPP.

2. $d U=0$

If $d U=0, U_{k}=U_{M}$, then it only needs to change the $I$. Let the step-size scaling factor be $\alpha(\alpha=0.0001)$, the disturbance step length be $\Delta l$, and the time step of the disturbance be $\Delta l=\alpha d I$, then

$$
I(k+1)=I(k)+\Delta l=I(k)+\alpha d I
$$

The sign of $d I$ represents the direction of disturbance of the system: if $d I<0$, then take the disturbance to the left, called as negative disturbance and if $d I>0$ then take the disturbance to the right, called as positive disturbance

\section{3. $d U \neq 0$}

- When $|d P / d U| \leq e$, as shown in Fig. 3: E-F segment, the MPP from this area search closer, so the use of smaller acceleration, the disturbance slowly to the MPP. Let the step-size scaling factor be $\beta$ $(\beta=0.1 \alpha)$ and the perturbation step be $\Delta l$, then

$$
\left\{\begin{array}{c}
\Delta l=\beta \sqrt[2]{|d I /(d U * U(k))|} \\
U(k+1)=U(k)+\Delta l
\end{array}\right.
$$

- When $|d P / d U| \geq e$, as shown in Fig.3: B-E segment and $\mathrm{F}-\mathrm{C}$ segment, these two regions are far away from MPP. Therefore, the disturbance speed is increased. Let the step-size scaling factor be $\lambda$ $(\lambda=0.25 \alpha)$ and the disturbance step be $\Delta l$, then

$$
\left\{\begin{array}{c}
\Delta l=\lambda \sqrt[4]{|d I /(d U * U(k))|} \\
U(k+1)=U(k)+\Delta l
\end{array}\right.
$$

$$
\begin{aligned}
& \text { As }|d I /(d U * U(k))|<1, \\
& \begin{aligned}
|d I /(d U * U(k))|<\sqrt[2]{|d I /(d U * U(k))|} \\
<\sqrt[4]{|d I /(d U * U(k))|}
\end{aligned}
\end{aligned}
$$

Thus, the overall tracking speed of the system is improving.

4. Direction of disturbance selection

- If $d I / d U>-I(k) / U(k)$, then $U(k)<U_{M}$, the search in the left side of the MPP, so the disturbance to the right side.

- If $d I / d U<-I(k) / U(k)$, then $U(k)>U_{M}$, the search has crossed the MPP, so the disturbance in the opposite direction.

\subsection{Genetic Algorithm}

The GA is a kind of intelligent bionic algorithm. In many intelligent optimization algorithms, GA has a good global search ability, and the convergence is good and the robustness is high [16]-[18]. In this paper, GA is used to establish the initial search range by the variable acceleration perturbation method in $\mathrm{A}-\mathrm{B}$ and $\mathrm{C}-\mathrm{D}$ segments, which reduces the tracking time of the system. Variables $S, T$ and $U$ are the input of GA and the output is D (duty cycle). Specific steps are as follows:

1. Initialization

Firstly, the output samples of the PV system are generated, the initial population is created in real-valued code and the population size $(N)$ is determined. Let the sampling power $P_{i}$ be taken as individual fitness, then the average fitness $F i t(\bar{P})$ and the maximum sampling power $P_{\max }$ are calculated. $P_{\max }$ is as the initial parents of the genetic search.

\section{Genetic manipulation}

- Selection: To avoid the premature convergence of GA, this paper uses the roulette method to initialize the individual population, and by the best retention strategy, the current most adaptable individuals are directly copied to the next generation. The individual roulette selection probability is:

$$
p_{i}=\frac{P_{i}}{N * F i t(\bar{P})}
$$


- Crossover: To improve the search ability of GA, this paper adopts the method of crossover operation to the individuals in the parents.

- Variation: To maintain the diversity of population, the amplification factor $A_{0}$ is introduced, and the result of the difference vector of any two individuals in the population is multiplied by $A_{0}$, and then added to $X^{i}(t)$ of the current $t$ generation. After the difference mutation, the individuals are:

$$
X^{i}(t+1)=X^{i}(t)+A_{0}\left(X^{j}(t)-X^{k}(t)\right)
$$

In the case of the external environment changes, the uniform population variation is used to generate the initial population.

3. Termination conditions

When the GA reaches the maximum number of iterations $\operatorname{Max} T$ or $|\Delta P|<\sigma$, the algorithm terminates the search.

\subsection{Overall Control Strategy}

The variable acceleration perturbation method based on GA of PV MPPT flow chart is shown in Fig. 4. First, the photovoltaic array is sampled to generate initial population and the initial conditions are set to calculate the sampling power $P_{i}(i=1,2 \ldots 10)$ as the fitness of individuals. Calculate $P_{\max }$ as the initial value of the genetic search and determine whether it could reach the maximum number of iterations or not. If the iteration reaches the maximum value, then accelerate the search to replace the disturbance of genetic search, otherwise still use genetic search. Search for several times when the disturbance changes power close to 0 , then the system reaches MPP. At this time, determine whether external environment changes, if it changes, then will be made uniform mutation operation of GA and let the algorithm generate the initial population; if the environment changes smaller, then using the retention of elite strategy, the elite will replace the individual parent to the search in the fitness of the worst individual.

\section{Simulation}

The simulation model is built up in Matlab/Simulink, and the MPPT submodule program is written in Matlab. The objective function is set to 2,500 , the population size is $N=30, \operatorname{MaxT}=20, P_{c}=0.9$, and $P_{m}=0.1$.

The light intensity decreases from $1,000 \mathrm{~W} / \mathrm{m}^{2}$ to $600 \mathrm{~W} / \mathrm{m}^{2}$ and then to $200 \mathrm{~W} / \mathrm{m}^{2}$ as shown in Fig. 5. The output power with time of the simulation results is shown in Fig. 6.

Figure 6(a) is the variable step perturbation method proposed in [19], while (b) is the variable acceleration perturbation method proposed in this paper. It can be seen that the tracking speed of (b) is significantly improved compared with (a) and the system oscillation is also significantly reduced near the MPP. Figure 6(c) is the variable acceleration perturbation method, which adds to the GA. Compared with (b), the tracking speed and the oscillation near the MPP of (c) are improved.

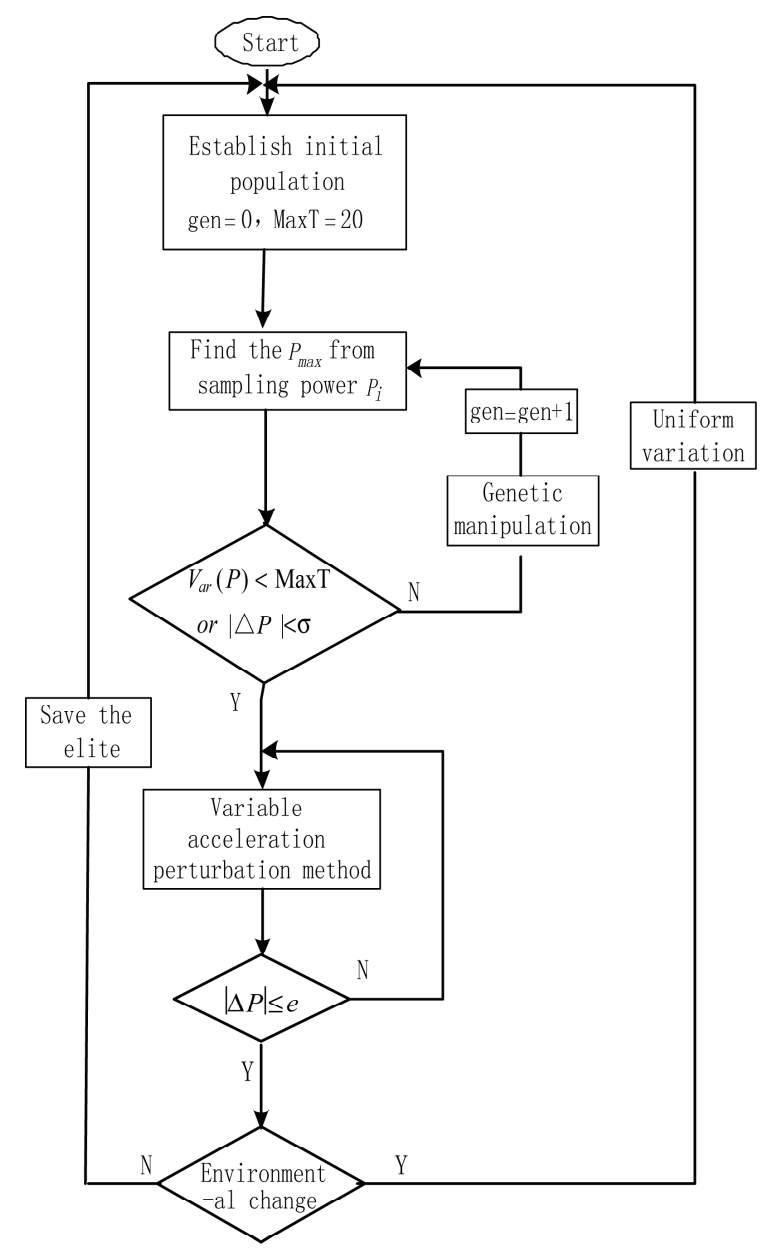

Figure 4. Flowchart of photovoltaic MPPT variable acceleration disturbance method based on GA.

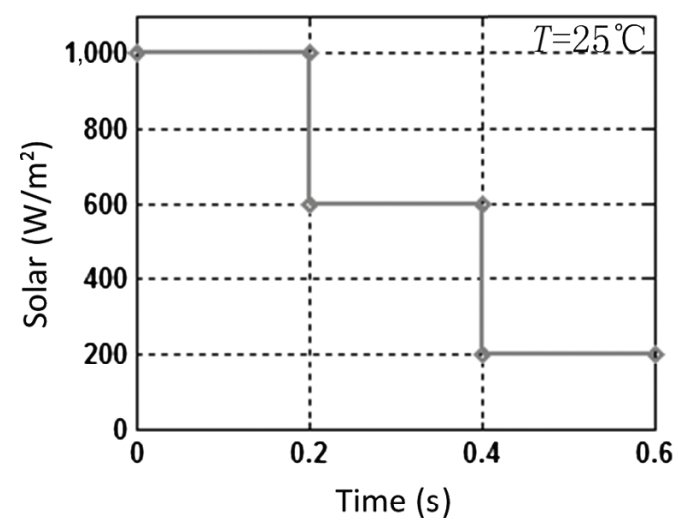

Figure 5. Solar curve.

When $S=600 \mathrm{~W} / \mathrm{m}^{2}, T$ increases from $15^{\circ} \mathrm{C}$ to $20^{\circ}$ and $20^{\circ} \mathrm{C}$ to $25^{\circ} \mathrm{C}$, and the power output of the simulation is shown in Fig. 7.

Figure $7(\mathrm{a})$ is variable step size perturbation method (b) is the variable acceleration disturbance method and, (c) is the variable accelerating perturbation method based on GA. From (a) and (b), it can be seen that the tracking speed of variable step size perturbation method is the most slowest, but the oscillation is very small, while the tracking speed of (b) is very quick, but the oscillation is quite big. 


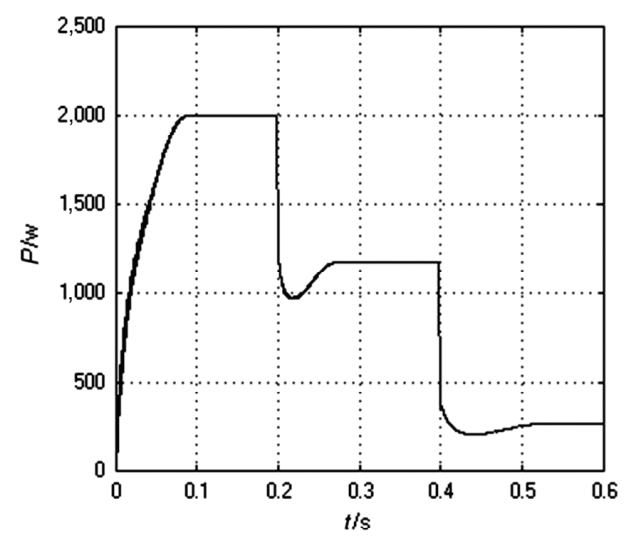

(a)

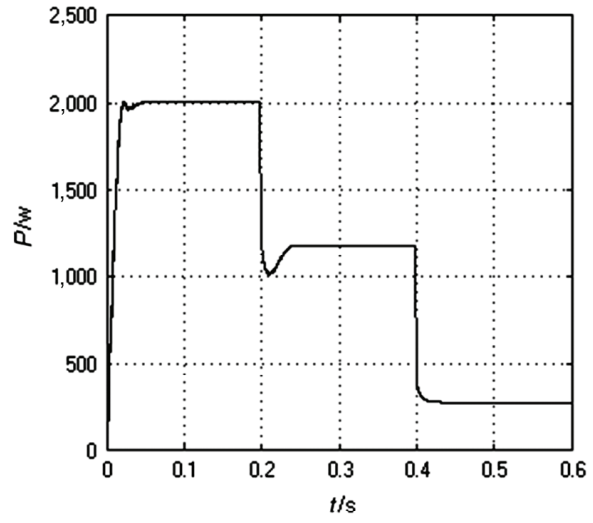

(b)

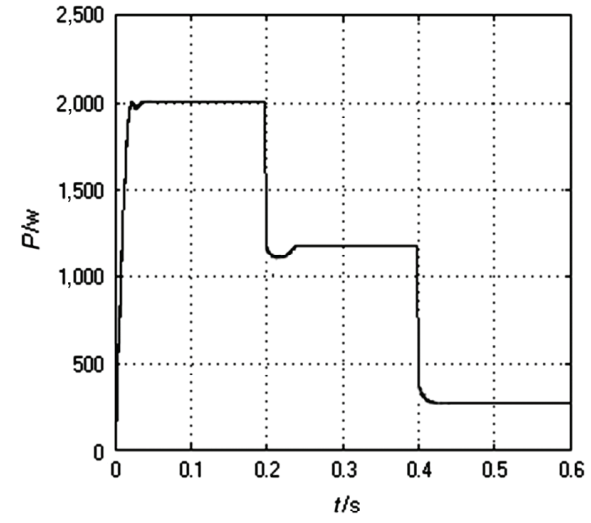

(c)

Figure 6. Output power under different control method. (a) Variable step size perturbation MPPT; (b) variable acceleration perturbation MPPT; and (c) variable acceleration disturbance MPPT based on GA.

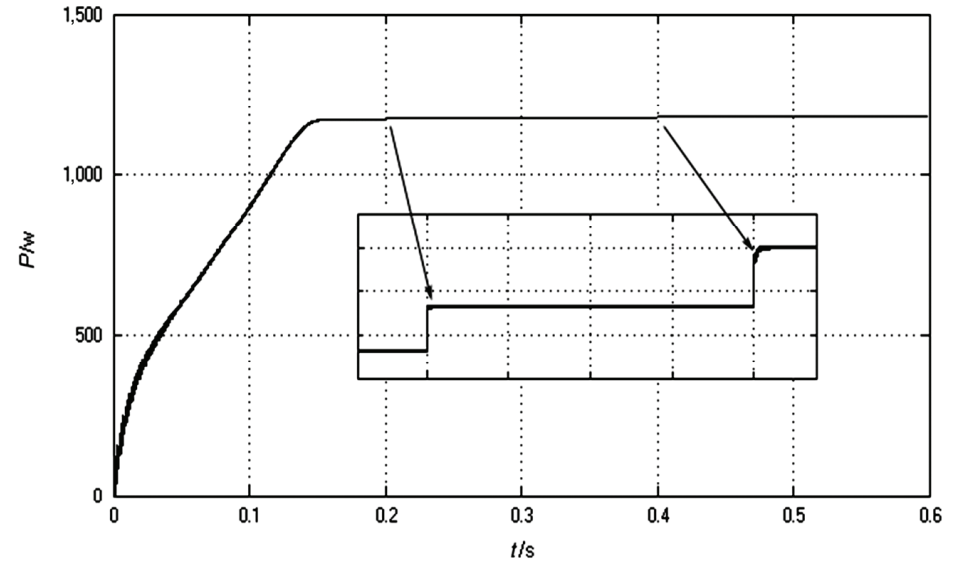

(a)

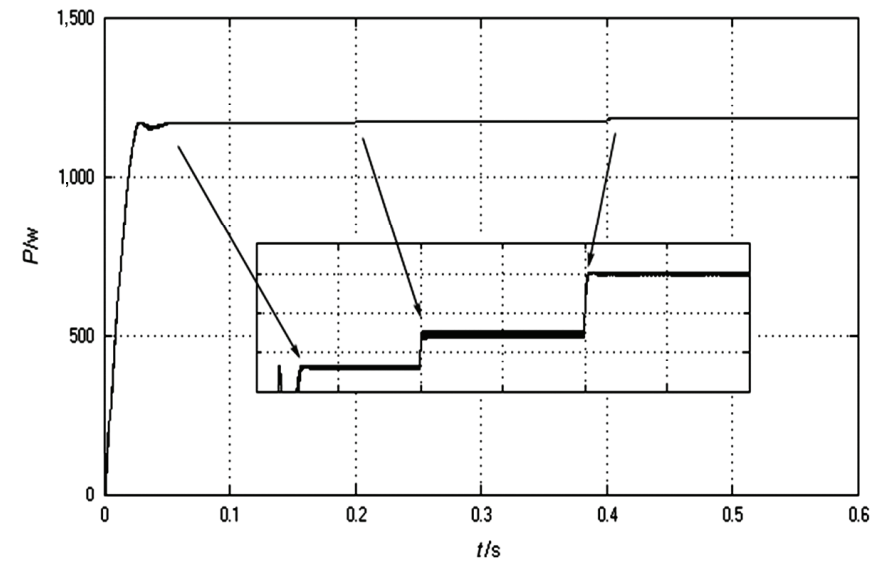

(b)

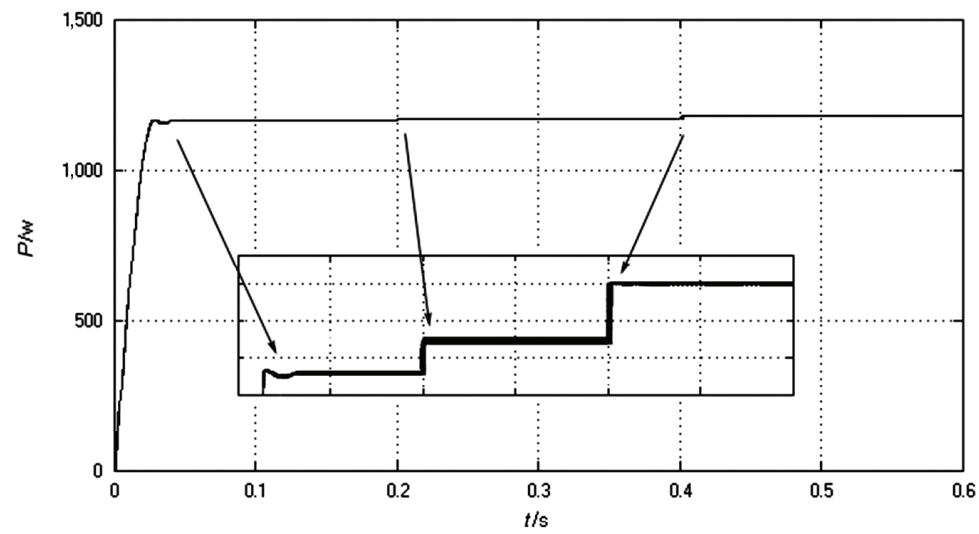

(c)

Figure 7. Output power at different temperature. (a) Variable step size perturbation MPPT; (b) variable acceleration perturbation MPPT; and (c) variable acceleration disturbance MPPT based on GA.

However, both small oscillation and quick tracking speed are shown in (c).

\section{Conclusion}

Based on the analysis of photovoltaic cell models, characteristics and advantages and disadvantages of various perturbations, a variable accelerating perturbation method based on the GA is proposed in this paper. Using Matlab/Simulink software to build the disturbance device, the GA-based accelerating perturbation strategy is simulated, and the simulation results show the following:

1. It has good adaptability when the environment changes abruptly and can track the MPP quickly and accurately. 
2. It has improved the problem that the GA cannot make the system work stable at the MPP, and the perturbation method at the MPP and the problem of poor adaptability to the environment.

\section{Acknowledgement}

This thesis is supported by the National Natural Science Foundation of China (61673165), the natural science foundation of Hunan (2017JJ4024), the Hunan provincial education department open fund (15k036) and the Hunan Key Laboratory (2016TP1018).

\section{References}

[1] R. Ni, D. Zheng F. Xu, and B. Cui, Simulation study on PV array output characteristics, Power Electronics, 47(3), 2013, 33-35.

[2] Y. Yang, B. Zhu, F. Zhao, et al., An adaptive and variable step MPPT method based on current predictive controllers, Proceedings of the CSEE, 34(6), 2014, 855-862.

[3] B. Wan, S. Luo, and L. Kang, PV MPPT based on duty cycle perturbation control and its simulation, Renewable Energy Resources, 31(10), 2013, 5-9.

[4] Y. Chen, J. Zhou, J. Li, and L. Zhou, Application of gradient variable step size MPPT algorithm in photovoltaic system, Proceedings of the CSEE, 39(17), 2014, 3156-3161.

[5] J. Gao and G. Li, Research on MPPT algorithm in combination with open circuit voltage and short circuit current, Power System Protection and Control, 43(24), 2015, 96-101.

[6] A.R. Reisi, M.H. Moradi, and S. Jamasb, Classification and comparison of maximum power point tracking techniques for photovoltaic system: A review, Renewable and Sustainable Energy Reviews, 19, 2013, 433-443.

[7] Y. Yang and K. Zhou, Photovoltaic cell modeling and MPPT controller strategies, Transactions of China Electrotechnical Society, 26(9), 2011, 2229-2234.

[8] H. Hang, W. Yang, and W. Zhu, Analysis and improvement of MPPT disturbance observer method for PV system, Power System Protection and Control, 42(9), 2014, 110-114.

[9] K.L. Lian, J.H. Jhang, and I.S. Tian, A maximum power point tracking method based on perturb-and-observe combined with particle swarm optimization, IEEE Journal of Photovoltaics, 2(4), 2014, 626-633.

[10] L. Shanshou, Z. Xing, Z. Hongkai, et al., Global MPPT method based on power closed-loop control and PSO algorithm, Proceedings of the CSEE, 2014, 34(28), 4809-4816.

[11] A.A.S. Mohamed, A. Berzoy, and O.A. Mohammed, Design and hardware implementation of FL-MPPT controller of PV systems based on GA and small-signal analysis, IEEE Transactions on Sustainable Energy, 1(8), 2017, 279-290.

[12] J. Fei and S. Wang, Feedback linearization-based adaptive fuzzy sliding mode control of MEMS triaxial gyroscope, International Journal of Robotics \&3 Automation, 28(1), 2013, 72-80.

[13] C.E.G. Cena, M. Artigas, R. Aracil, and R.J. Saltarén, Optimal analysis of a teleoperated system with an adaptative controller, International Journal of Robotics $\&$ Automation, 26(26), 2011, 271-311.

[14] J. Yang and L. Zhu, Research on photovoltaic cell model based on Matlab/Simulink, Modern Electronics Technique, 34(24), 2011, 192-195.

[15] S. Kaitwanidvilai, P. Olranthichachat, and I. Ngamroo, Weight optimization and structure specified robust H\# loop-shaping control of a pneumatic servo system using genetic algorithm, International Journal of Robotics $\&$ Automation, 25(3), 2010, 229-239.

[16] T. Ababsa, N. Djedl, and Y. Duthen, Genetic programmingbased self-reconfiguration planning for metamorphic robot, International Journal of Automation \& Computing, 2017, 1-12.

[17] F. Piltan, A. Jalali, and N. Sulaiman, Design of PC-based sliding mode controller and normalized sliding surface slope using
PSO method for robot manipulator, International Journal of Robotics \& Automation, 2(4), 2011, 101-114.

[18] P. Jha, A neural network approach for inverse kinematic of a SCARA manipulator, International Journal of Robotics 83 Automation, 3(1), 2014, 31-40.

[19] D. Zhou and Y. Chen, Maximum power point tracking strategy based on modified variable step-size incremental conductance algorithm, Power System Technology, 39(6) 2015, 1491-1498.

[20] A. Safari and S. Mekhilef, Simulation and hardware unification of incremental conductance MPPT with direct control method using Ćuk converter, IEEE Transactions on Industrial Electronics, 58(4), 2011, 1154-1161.

[21] L. He and S. Cheng, MPPT algorithm based on genetic algorithm and perturbation observation method, Modern Electronics Technology, 24(31), 2009, 199-202.

[22] L. Chouksey, P. Akash Pattanaik, and R.K. Saket, Maximum power point tracking of photovoltaic system using two input and two output fuzzy system, Lecture Notes in Electrical Engineering, 326, 2015, 245-253.

[23] T. Radjai, L. Rahmani, S. Mekhiief, et al., Implementation of a modified incremental conductance MPPT algorithm with direct control based on a fuzzy duty cycle change estimator using dSPACE, Solar Energy, 110, 2014, 325-337.

[24] C. Liu, J. Chen, Y. Liu, et al., An asymmetrical fuzzylogic-control-based MPPT algorithm for photovoltaic systems, Energies, 7(4), 2014, 2177-2193.

[25] R. Rajesh and M. Mabel, Efficiency analysis of a multi-fuzzy logic controller for the determination of operating points in a PV system, Solar Energy, 99, 2014, 77-87.

[26] A.K. Abdelsalam, A.M. Massoud, S. Ahmed, et al., Highperformance adaptive perturb and observe MPPT technique for photovoltaic-based microgrids, IEEE Transactions on Power Electronics, 26(4), 2011, 1010-1021.

[27] B. Parida, S. Iniyan, and R. Goic, A review of solar photovoltaic technologies Renewable and Sustainable Energy Reviews, 15(3), 2011, 1625-1636.

[28] K. Sundareswaran, V. Vignesh Kumar, and S. Palani, Application of a combined particle swarm optimization and perturb and observe method for MPPT in PV systems under partial shading conditions, Renewable Energy, 75, 2015, 308-317.

[29] H. Rezk and A. Eltamaly, A comprehensive comparison of different MPPT techniques for photovoltaic systems, Solar Energy, 112, 2015, 1-11.

[30] Y. Chen, Z. Lai, and R. Liang, A novel auto-scaling variable step-size MPPT method for a PV system, Solar Energy, 2014, 102, 247-256.

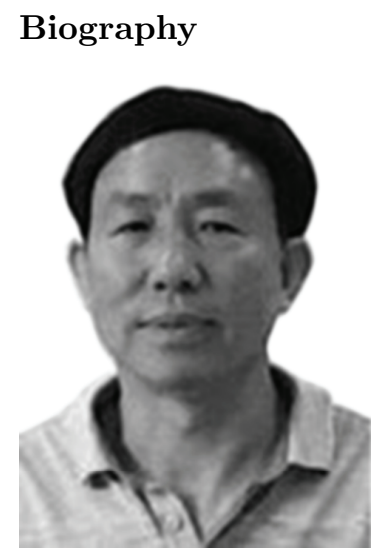

Shengqing $L i$ graduated in electrical theory and new technology from the Hunan University and received a doctorate in June 2005. Mainly engaged in electrical engineering teaching and research work, the research direction for the distribution network power quality control, power electronic system control, new energy distributed power generation and micro-grid control technology. In recent years, presided over the completion of the national self-fund project, the national science and technology enterprise innovation fund projects and provincial and ministerial level important projects more than 20, to participate in the completion of the national 863 plan 1. 


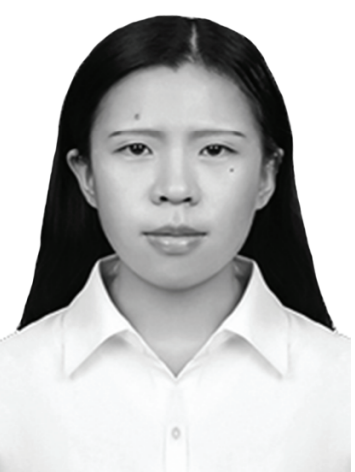

Wenfeng $W u$, third year graduate student, is studying in the School of Electrical and Information Engineering, Hunan University of Technology, Zhuzhou, Hunan, China. She is majoring in power system and its automation. Her research direction is photovoltaic power generation.

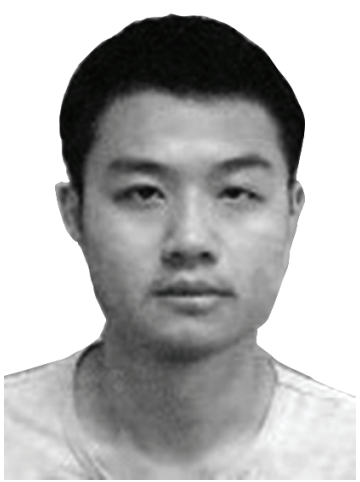

Dinghuan $M a$, third year graduate student, is studying in the School of Electrical and Information Engineering, Hunan University of Technology, Zhuzhou, Hunan, China. He is majoring in electrical engineering. His research direction is microgrid energy storage. 